\title{
Response variability in pigeons in a Pavlovian task
}

\author{
W. DaVid STAHLMan \\ University of California, Los Angeles, California \\ Michael E. Young \\ Southern Illinois University, Carbondale, Illinois \\ AND \\ Aaron P. BLAisdell \\ University of California, Los Angeles, California
}

\begin{abstract}
Instrumental response variation is inversely related to reward probability. Gharib, Derby, and Roberts (2001) theorized that individuals behave more variably when their expectation of reward is low. They postulate that this behavioral rule assists the discovery of alternative actions when a target response is unlikely to be reinforced. This suggests that response variability may be unaffected in a situation in which an animal's behavior is inconsequential to outcome delivery. We trained 6 pigeons in a within-subjects Pavlovian autoshaping procedure. On any given trial, the pigeons were presented with one of six colored discs on a touchscreen; each stimulus was associated with a particular probability of food, ranging from $100 \%$ to $0.6 \%$. Pecking was more variable with low probabilities of food delivery, thus extending the rule relating variability and expectation to a Pavlovian situation.
\end{abstract}

Behavioral variation increases markedly during extinction procedures. When previously reinforced behavior is no longer rewarded, the strength of behavior is not solely affected; the behavior itself tends to become more variable in nature. Page and Neuringer (1985) referred to this as "respondent variability" (p. 21). Although many researchers have noted this pattern (Antonitis, 1951; Balsam, Deich, Ohyama, \& Stokes, 1998; Eckerman \& Lanson, 1969; Herrick \& Bromberger, 1965; Millenson \& Hurwitz, 1961; Neuringer, Kornell, \& Olufs, 2001; Notterman, 1959; Stebbins \& Lanson, 1962), few had examined the underlying nature of this relationship in detail until recently. Extinction differs from acquisition in a number of important ways, including (but not limited to) levels of reward density, frustration effects, and expectation of reinforcement. Although researchers have noted that extinction increases variability and have long considered this relationship theoretically important, few have investigated the reason(s) for which extinction increased behavioral variability.

Gharib, Derby, and Roberts (2001) trained rats on the fixed-interval (FI) peak procedure; on $80 \%$ of trials, rats were reinforced with a food pellet following the first response after $40 \mathrm{sec}$ from the start of the trial (i.e., FI-40 sec). The remaining trials were nonreinforced probes, which lasted $195 \mathrm{sec}$ and terminated without food reinforcement. The variability of rats' barpress durations increased significantly following the point on probe trials at which food was expected but not delivered (i.e., after $40 \mathrm{sec}$ on a trial).
These results led Gharib et al. (2001) to propose a rule: Reduction in reward expectation increases the variation in the form of behavior.

Gharib, Gade, and Roberts (2004) directly tested this rule using a discrete-trials procedure with two discriminative stimuli (DS): light and white noise. Each barpress during a DS had a $25 \%$ probability of terminating the trial. Trials with one DS (e.g., light) always terminated with reinforcement, whereas trials with the other DS (e.g., white noise) ended with reinforcement only $25 \%$ of the time. Barpress durations were more variable on trials with the DS that signaled a lower probability of reward. They concluded that reduced reward expectation produces greater variability in responding.

Recent evidence suggests that these effects are general and not limited to a specific species, domain (e.g., temporal), or behavioral requirement (e.g., barpressing). Stahlman, Roberts, and Blaisdell (2010) extended the Gharib et al. (2001; Gharib et al., 2004) results by investigating instrumental touchscreen pecking in pigeons. Pigeons were reinforced with mixed grain for pecking at a set of colored discs. On any given trial, the pigeons were randomly presented with one of six discs, each a different color. Each color was associated with a particular probability of reinforcement, ranging from $100 \%$ to $0.6 \%$, delivered at the termination of the trial. Each peck to the disc had a $20 \%$ chance of ending a trial. Pecks to discs less predictive of reward exhibited greater spatiotemporal variation than did pecks to discs more predictive of reward. Thus, the expec-

A. P. Blaisdell, blaisdell@psych.ucla.edu 
tation of reward determined the degree of variation in both spatial and temporal aspects of behavior.

All of the above studies were focused on the variations of operant responses. By definition, in an operant paradigm, the delivery of reward depends on instrumental action (e.g., barpressing). Gharib et al. (2004) couched the theoretical implications for the rule squarely in goaldirected terms:

If an animal's actions vary too little, it will not find better ways of doing things; if they vary too much, rewarded actions will not be repeated. So at any time there is an optimal amount of variation, which changes as the costs and benefits of variation change. Animals that learn instrumentally would profit from a mechanism that regulates variation so that the actual amount is close to the optimal amount. (p. 271)

Instrumental conditioning requires the selection of an action from numerous alternative behavioral options. Action is selected through differential reinforcement. As the likelihood of reinforcement decreases, the selective force governing instrumental behavior may relax, resulting in more variety in behavior. This is a potential mechanism for the negative relationship between reward expectation and variability in behavior.

Does reward expectancy also affect behavioral variation in a Pavlovian conditioning preparation? By definition, Pavlovian responses have no bearing on reinforcement delivery. For example, a rat's conditioned fear response to a cue that signals the delivery of shock does not prevent the shock from being delivered. Similarly, a pigeon will peck at a keylight that signals the delivery of grain in an autoshaping task, even though the pigeon is not required to peck the keylight in order to obtain the grain (Brown \& Jenkins, 1968). This effect is perhaps most dramatic if we consider a situation in which the pigeon is placed on an omission schedule (also referred to as negative automaintenance); the pigeon will persist in pecking at the target, despite the fact that this action prevents the delivery of food (Williams \& Williams, 1969). Pavlovian responses are elicited, but not selected for, by the contingencies of stimuli in the environment. It seems that the relationship between signaled reward likelihood and behavioral variation might not be applicable to a Pavlovian situation, where behavior is inconsequential to the delivery of food.

We conducted an experiment to investigate this issue and to further test the Gharib et al. (2004) rule. On any given trial, a pigeon was presented with one of six colored circles on a touchscreen, each associated with a specific probability of grain delivery at the end of the trial. All trials terminated after $10 \mathrm{sec}$. Responses to the screen had no effect on the probability of grain delivery. We found that pigeons responded with greater spatiotemporal variability on trials during which food was unlikely to be delivered.

\section{METHOD}

\section{Subjects}

One male White Carneaux and 5 male racing homer pigeons (Columba livia; Double T Farm, Iowa) served as subjects. Four birds had previously been utilized in a simple go/no-go visual discrimination task; the other 2 birds had been subjects in a spatial blocking task. These birds were selected for use because these previous training experiences would likely have no appreciable effect with respect to the present manipulation, since the experimental stimuli in the prior studies were very different from those used in this experiment.

The pigeons were maintained at $80 \%-85 \%$ of their free-feeding weight. They were individually housed with a 12:12-h light:dark cycle and had free access to water and grit. The experiment was conducted during the light portion of the cycle.

\section{Apparatus}

Testing was done in a flat-black Plexiglas chamber $(38 \times 36 \times$ $38 \mathrm{~cm}$ ). Stimuli were generated on a color monitor (NEC MultiSync LCD1550M), visible through a $23.2 \times 30.5 \mathrm{~cm}$ viewing window in the middle of the front panel of the chamber. The stimuli consisted of red, blue, white, yellow, green, and magenta circular discs, $5.5 \mathrm{~cm}$ in diameter. At the center of each disc was a small black dot $(2 \mathrm{~mm}$ in diameter). The bottom edge of the viewing window was $13 \mathrm{~cm}$ above the chamber floor. Pecks to the monitor were detected by an infrared touchscreen (Carroll Touch, Elotouch Systems, Fremont, CA) mounted on the front panel. A $28-\mathrm{V}$ houselight located in the ceiling of the chamber was on at all times. A food hopper (Coulbourn Instruments, Allentown, PA) was located in the center of the front panel, its access hole flush with the chamber floor. Experimental events were controlled and recorded with a Pentium III computer (Dell, Austin, TX). A video card controlled the monitor in SVGA graphics mode $(800 \times 600$ pixels $)$. The resolution of the touchscreen was 710 horizontal $\times 644$ vertical units.

\section{Procedure}

A trial consisted of the presentation of one of the six colored discs in the center of the computer touchscreen. Each color was randomly selected and was equally likely ( $16.7 \%$ chance) to occur on any trial. Trials were terminated after $10 \mathrm{sec}$, at which time a 60 -sec intertrial interval (ITI) began. For each bird, individual colored discs were associated with a specific probability of unconditioned stimulus (US) delivery at the termination of a trial. For example, a bird may have been trained with the following color-probability assignments: red, $100 \%$ (i.e., food delivered after every red trial); green, $35.3 \%$; blue, $12.5 \%$; white, $4.4 \%$; magenta, $1.1 \%$; and yellow, $0.6 \%$. These values were chosen because they had previously been shown to be effective in demonstrating expectation-driven differences in variation in an instrumental task (Stahlman et al., 2010). The US consisted of raising and illuminating the grain hopper for $3 \mathrm{sec}$ at the beginning of the ITI. The screen coordinates and session time of each peck were recorded. Color assignments were randomized across subjects. Each session lasted $60 \mathrm{~min}$. There were 180 sessions, with 51 trials per session.

\section{Measures}

We calculated the median spatial location of responses separately for each pigeon on each trial type. The median $x$ position and $y$ position were used to eliminate the effects of extreme values; because the $x$ and $y$ spatial positions had different distributions, using the median was a conservative choice, and we thus avoided using different transformations for the two axes. The touchscreen recorded discrete (i.e., noncontinuous) spatial locations of pecks; to make the data continuous, we added a small random value to the $x$ and $y$ values of each response. This method, called jittering, is commonly utilized to break ties in discrete measures. ${ }^{1}$

We calculated the Euclidean distance between a bird's median peck location and each peck's location for that bird. Thus, if a bird's responses were tightly clustered anywhere on the display, the distances of each peck from this median would be small, reflecting low spatial variability. The opposite is true for highly variable pecking.

We performed a similar procedure with respect to measuring temporal variability. We first calculated the mean logarithm of the interresponse time (IRT) for each bird on each trial type (IRTs were 
well described by a log normal distribution). The absolute difference between each individual IRT and the mean IRT for each bird is our measure of IRT variability. Because of large differences in overall response rates across trial types, we used the restricted maximum likelihood (REML) estimation method for our statistical tests. Unlike conventional maximum likelihood estimation methods, the REML method produces unbiased parameter estimates. For this reason, the REML method of estimation is superior to the traditional expected mean squares method for highly unbalanced designs (Pinheiro \& Bates, 2004). We also examined within-sessions effects by dividing individual sessions into ten 6-min bins.

We were primarily interested in the postacquisition effects of reward probability on behavior. Early in training, the pigeons would not have learned the differential payoffs across stimuli, and therefore the inclusion of early sessions would have been uninformative. Therefore, we report data collected from the last 76 sessions, which indicate stable postacquisition behavior.

\section{RESULTS}

\section{Response Rates}

A repeated measures ANOVA of the number of pecks (on or off the disc) as a function of disc probability and bin revealed a main effect of probability $[F(5,27)=4.37, p<$ $.01]$ but no other significant effects $(p \mathrm{~s}>.4)$. The peck rate varied logarithmically from 7.5 pecks per bin for the $100 \%$ payoff disc to 2.8 pecks per bin for the $.6 \%$ payoff $\operatorname{disc}[t(27)=4.38, p<.001]$.

\section{Spatial Variability}

We found a significant effect of US probability on the spatial variability of responses $[F(5,23)=10.72, p<$ $.001]$ (see Figure 1). As was expected, we discovered a significant linear contrast for US probability $[t(24)=$ $7.12, p<.01]$. This effect was consistent across all subjects. Figure 1 demonstrates that the pigeons responded with increasing spatial variability as the probability of grain decreased. Figure 2 shows nonparametric density plots for each trial type, collapsed across all of the birds. Varying the probability of grain had much stronger effects

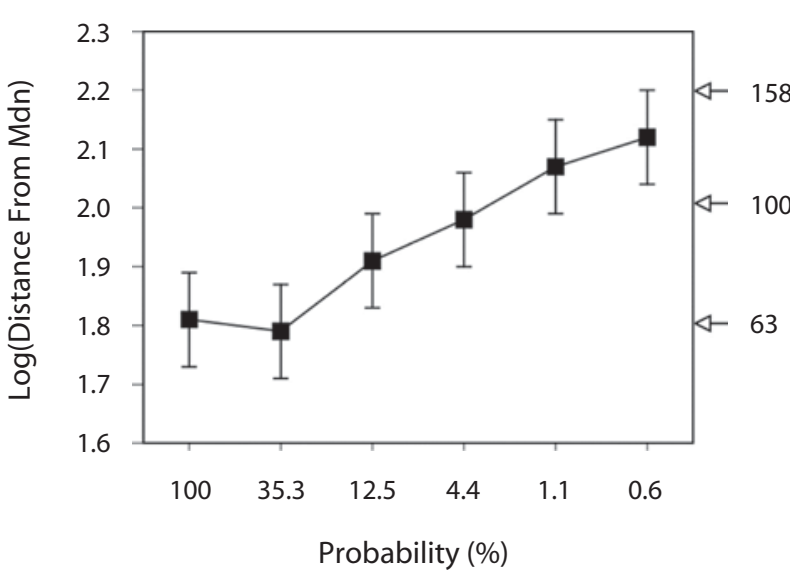

Figure 1. Graph of the log of the mean distance (in pixels) from the individual bird median spatial location as a function of reward probability. The raw values of the dependent variable are located on the right-side vertical axis. Error bars denote standard errors of the means. along the horizontal than along the vertical dimension of the screen.

It has been suggested that differences in response rates across trial types may account for this effect on variability. The effect of reinforcement on spatial variability cannot be explained as a function of response rate; linear mixed effects analyses of the slope of the relation between the mean IRT as a predictor of variability revealed no relation between peck rate and spatial variability $[F(1,5)=0.84$, $p>.25]$.

The pigeons tended to increase the amount of spatial variability over the course of a session, from 81 pixels in the first bin to 102 pixels in the last, but this increase was uniform across all trial types. We found a significant effect of within-sessions time bin on the level of variability $[F(9,38)=3.38, p<.01]$. A linear contrast for bin was significant $[t(43)=4.62, p<.01]$. There was no probability $\times$ bin interaction, however $[F(45,131)=1.21$, $p>.05]$.

We discovered that the within-trials spatial variability of responses tended to decrease approaching the end of the trial $[F(1,5)=47.78, p<.01]$ (see Figure 3). Time in trial interacted with bin $[F(9,75)=3.09, p<.01]$; the interaction was due to spatial variability decreasing more rapidly within trials during the first $12 \mathrm{~min}$ (i.e., the first two bins) than it did during the rest of the session. There was no probability $\times$ time in trial interaction.

\section{Temporal Variability}

We found a significant effect of US probability on the temporal variability of responses $[F(5,25)=2.87, p<$ $.05]$ (see Figures 4 and 5). A planned contrast revealed a significant linear trend for US probability $[t(28)=3.41$, $p<.01]$. Figure 4 shows that temporal response variability increased with decreasing food probability. Interestingly, this increase in variability was obtained without a significant increase in the mean log IRTs with lower probability of the US. The standard deviation parameter, $s$, of the best-fitting log normal probability distribution was greater for the $\leq 4.4 \%$ keys $(95 \% \mathrm{CI}=.353, .370)$ than for the $\geq 12.5 \%$ keys $(95 \% \mathrm{CI}=.322, .328)$. Figure 5 demonstrates the flatter, wider probability distribution of IRTs on the three lowest-rewarded trials, relative to the other, more frequently rewarded trials.

In contrast to the spatial data analysis, there was no significant effect of bin on the temporal variation of responding $[F(9,66)=1.62, p>.05]$. Also, we did not find an effect of time in trial on IRT variability, although the trend was in the expected direction $[F(5,21)=1.30$, $p>.05]$. Finally, unlike that in the spatial data analysis, the effect in temporal variability may be explainable in terms of response rate differences across trial types; linear mixed effects analyses revealed a positive relation between mean IRT and temporal variability $[F(1,5)=$ $18.81, p<.01]$.

A particularly striking effect of reward probability is demonstrated in Figure 6. When US delivery was at least $35.3 \%$, responses clustered toward the end of the trial. As the US became less likely, this tendency became weaker and even reversed at the lowest probability of food, so that 

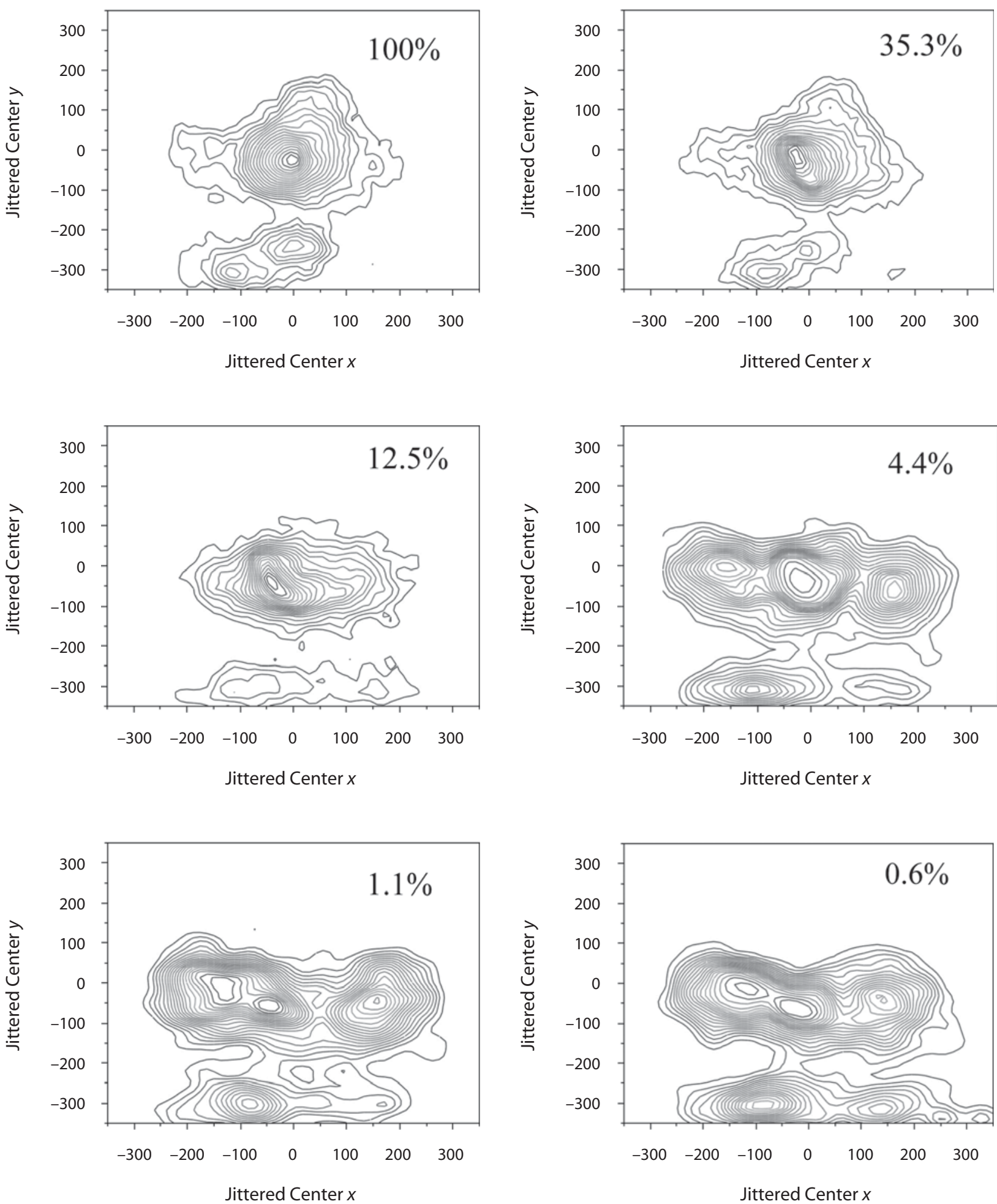

Figure 2. Peck density plots for each of the six trial types, collapsed across subject. The peck density plot models a smooth surface that describes how dense the data points are at each point in that surface and functions like a typographical map. The plot adds a set of contour lines showing the density in $5 \%$ intervals in which the densest areas are encompassed first. The JMP (Version 8, SAS Institute, Cary, NC) bivariate nonparametric density function was used to generate these plots. 


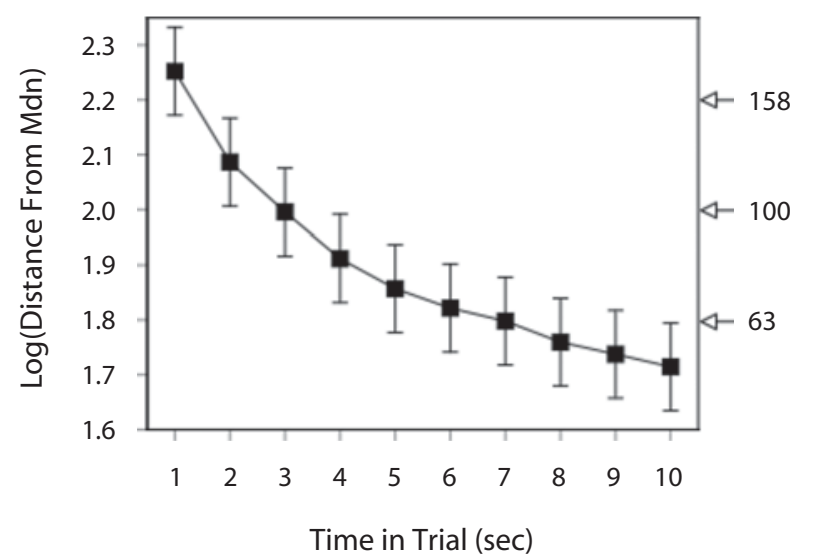

Figure 3. Graph of the log of the mean distance (in pixels) from the individual bird median spatial location as a function of trial time. The raw values of the dependent variable are located on the right-side vertical axis. Error bars denote standard errors of the means.

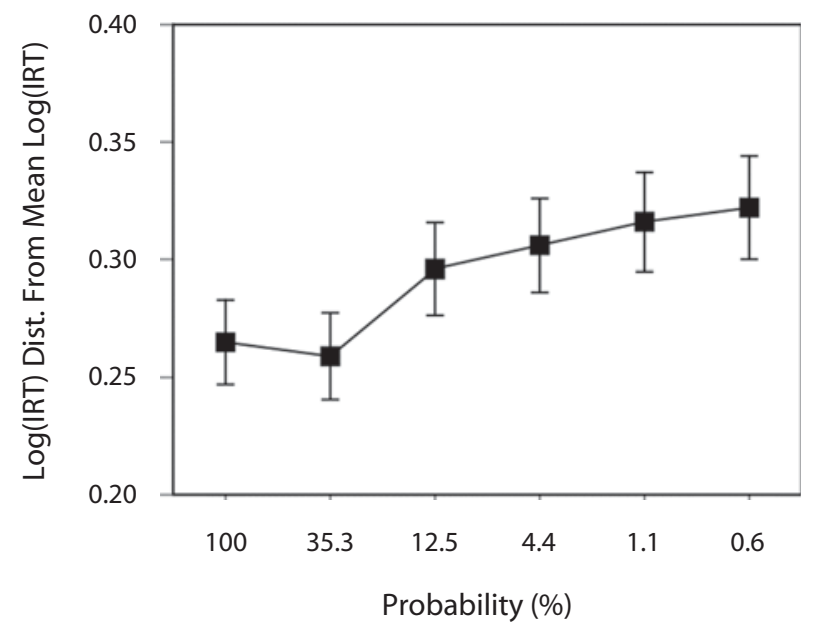

Figure 4. $\log (\mathrm{IRT})$ of temporal distance (in seconds) from the mean $\log ($ IRT) for each subject as a function of reward probability. Error bars denote standard errors of the means.

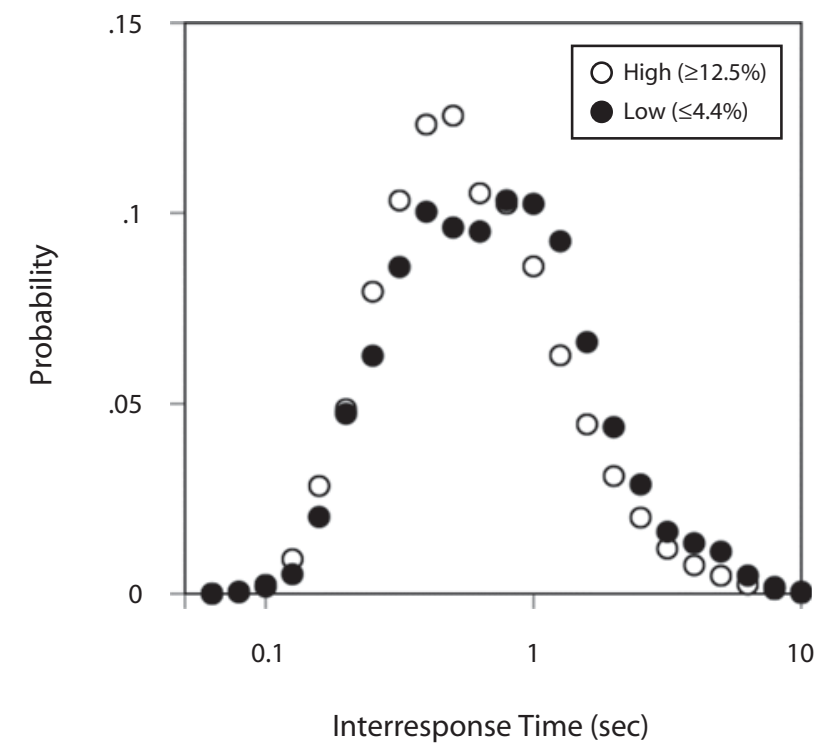

Figure 5. Response probability as a function of IRT (in seconds) on a logarithmic scale for the lower and higher probability stimuli (cf. Gharib et al., 2004).

the density of responses was greatest at the very beginning of the trial. A repeated measures ANOVA of time of peck within trial as a function of log-transformed outcome probability revealed differences in the timing of the pecks $[F(5,23)=2.97, p<.05]$, with a strong logarithmic trend from the richest schedule $(M=6.4 \mathrm{sec})$ to the leanest schedule $(M=4.6)[t(23)=3.59, p<.01]$.

\section{DISCUSSION}

Our results extend and clarify the relationship between reinforcement and behavioral variability. This relationship does not have to occur only in operant preparations; instead, the manipulation of a predictive relationship be- tween stimuli is sufficient to instantiate differences in respondent variability.

We discovered significant linear effects relating decreased US likelihood to high levels of spatial and temporal response variability in a Pavlovian task. This finding suggests that Pavlovian response variability is affected by outcome expectation in the same manner as instrumental variability. In addition, we found evidence that withinsessions factors affect variability. We will address both of these aspects in detail.

\section{Reinforcement Contingencies}

As was previously mentioned, the theoretical structure for the relationship between positive outcome expectation 


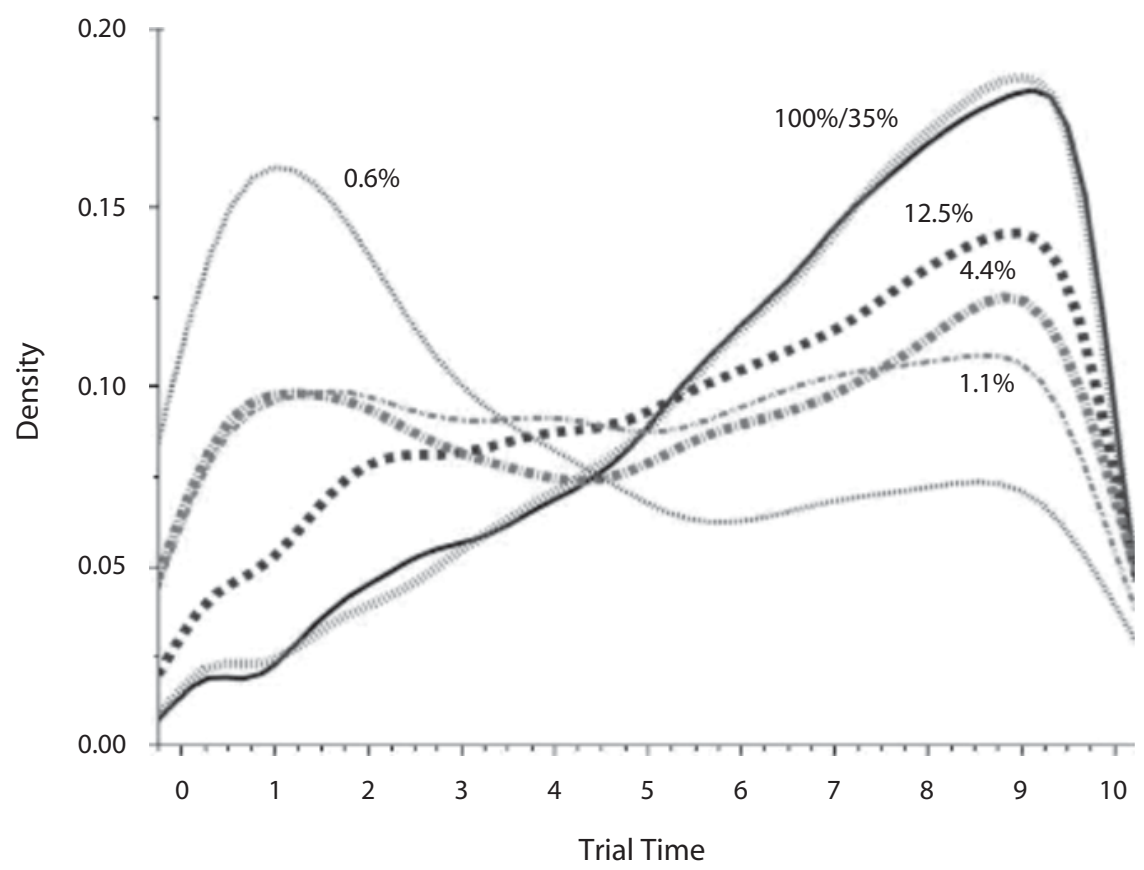

Figure 6. Density of pecks on each trial type as a function of trial time (in seconds), collapsed across subjects.

and behavioral variability has been expressed in purely instrumental terminology. Essentially, the relationship could serve as a mechanism for the optimization of behaviorfor example, perseverating on an operant when reward is plausible and exploring other behavioral options when reward is unlikely. This implies that respondent behavior on Pavlovian schedules may not be subject to the same modulation by US probability. Our data unequivocally reject this prediction. Indeed, we observe the very same pattern of behavior in a Pavlovian conditioning preparation as we have found in operant procedures in our lab (Stahlman et al., 2010).

The implications of these findings are somewhat less clear. There is a pair of interpretations of our data regarding the underlying nature of the behavior that we measured:

Adventitious reinforcement. Some researchers have asserted that autoshaping procedures may train responses akin to instrumental actions through adventitious reinforcement (Atnip, 1977). These researchers suggest that initial pecks are elicited but not selected for; the subsequent contiguity of pecks to grain delivery may contribute to incidental instrumental conditioning. This suggests that the pigeons' response variability in our task may have been controlled by the accidental contiguity between pecks and grain accessibility. Pigeons are visual foragers and learn through reinforcement contingencies during early development which items are or are not acceptable foods (Balsam et al., 1998). Thus, the contiguity between discs presented on the touchscreen and delivery of a food US may engage the feeding system in creating the illusion of an instrumental contingency between the two (e.g., Skinner, 1948), despite the lack of any actual contingency.
Predictive learning. It is possible that Pavlovian associations control modulated behavioral variability in associative preparations. This would make sense, because the relationship that we have elucidated seems to be largely predictive (i.e., Pavlovian) in nature. The degree to which animals can predict the delivery (or nondelivery) of food reinforcement modulates their behavior, irrespective of the contingency between their actions and a trial's outcome. This does not directly speak as a criticism of the idea that operant learning controls variability but does suggest that the modulation of form may be parsimoniously accounted for by Pavlovian learning. After all, instrumental conditioning preparations also encode Pavlovian, stimulus-stimulus relationships in experimental subjects (Rescorla \& Solomon, 1967; Spence, 1956; Trapold \& Overmier, 1972).

Although this experiment does not clearly give any way to elucidate the degree to which each of the above reasons contributes to our results, it does suggest future potential research. For example, it would be interesting to investigate the differences in behavioral variability across dimensions of reinforcement probability and schedule type (e.g., Pavlovian vs. omission schedules).

\section{Within-Sessions Factors}

Spatial response variability decreased within trials, so that variability was greatest at the beginning of the trial and progressively lessened approaching the end of the trial and the potential imminent delivery of the US (see Figure 3). Others have made similar reports that behavioral variation tends to decrease with approach of the time of reinforcement (Cherot, Jones, \& Neuringer, 1996; Gharib et al., 2001; Neuringer, 1991; Schwartz, 1982). Our replication of 


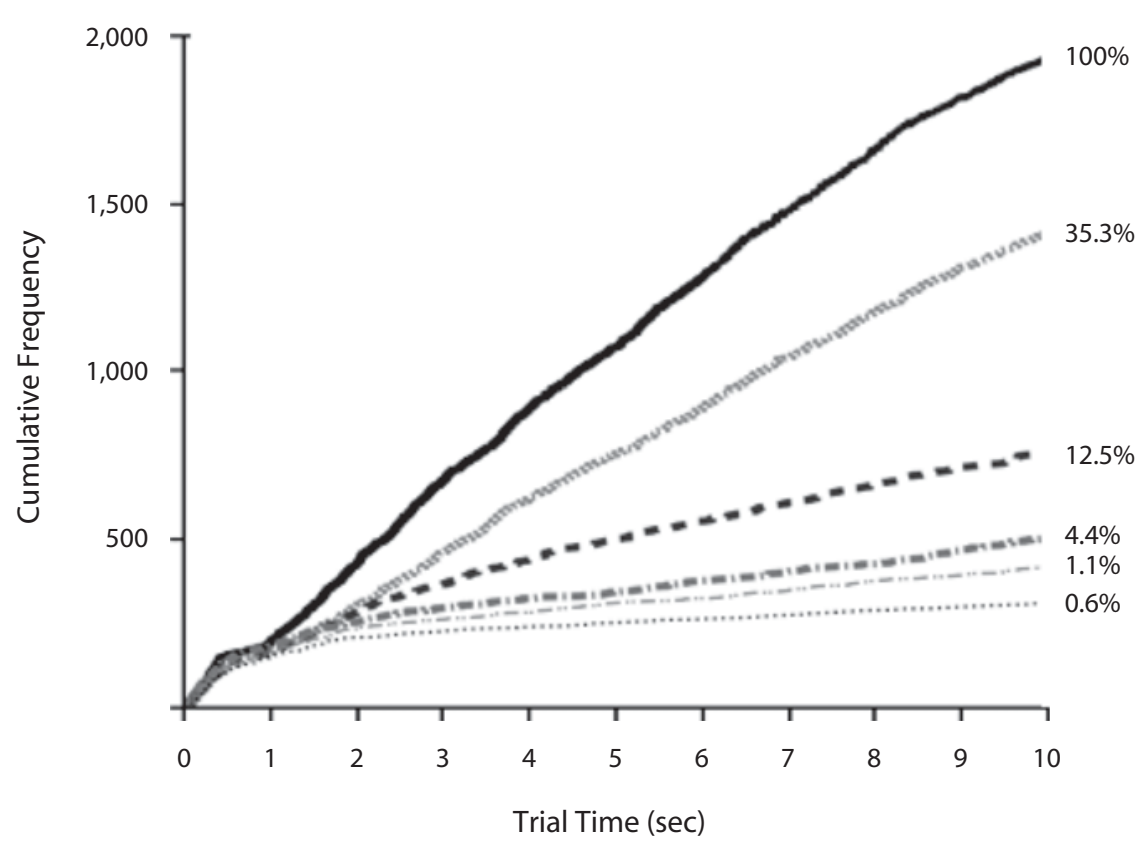

Figure 7. Cumulative frequency plot of first responses within trial type as a function of time in trial.

this general finding therefore provides cross-validation of our experimental procedure and further confirms the direct effect of outcome expectation on behavioral variation.

We found that spatial variability increased by $25 \%$ over the course of a session. This effect is likely due to a nonassociative factor, such as gradual satiety. The fact that the downward shift in within-trials variability decreased with session duration seems to fit with this hypothesis. If satiety is indeed the underlying cause of these effects, motivation should be an important factor to consider as a determinant of expectation-driven variation. This is unsurprising, because motivational effects are well documented in other crucial aspects of behavior, such as response rate (e.g., Bizo, Bogdanov, \& Killeen, 1998; DeMarse, Killeen, \& Baker, 1999).

\section{Stimulus Control of Behavior}

We noted an interesting effect with respect to stimulus control. Figure 6 indicates that high probabilities of US delivery tended to cluster responses toward the termination of the trial. This was expected: As the expectation of food grew during the course of a trial, so too did the likelihood of the pigeons' response. What may appear more difficult to explain is the density distribution of responses on trials associated with a low probability of US delivery. On the leanest trial type (i.e., $0.6 \%$ chance of food), responses were most likely at the very start of the trial. We believe these early responses to be reflective of pecking being controlled by trial onset; in other words, the very commencement of a trial was enough to trigger some responding. As the trial progressed, the discriminative cue (i.e., color) gained control of behavior. A cumulative frequency plot (Figure 7) of first responses within trial type demonstrates this effect: Although the pigeons were somewhat likely to commence responding at virtually any point on a high-probability (e.g., 100\%) trial, they tended to initiate responding in the first few seconds of a low-probability (e.g., $0.6 \%$ ) trial. If the pigeons did not respond at the very onset of a low-probability trial, they were unlikely to commence responding at a later point during the trial. This effect has obvious connections to well-known phenomena, such as inhibition of delay (Pavlov, 1927).

\section{Conclusions}

This study demonstrates that behavioral variability increases with decreasing likelihood of an appetitive outcome in a Pavlovian task. The mere arrangement of a predictive relationship between stimuli is enough to modulate behavioral variability and requires that no operant be performed by the organism. To our knowledge, this is the first time that this effect has been documented. These findings suggest that previous theoretical positions, which posit an instrumental focus on the generation of variability, may be insufficient. We also presented evidence that suggests that certain nonassociative factors play a role in the generation of behavioral variability. This work sheds new light on the nature of reinforcement and variability and suggests a course of research for continuing to investigate the underlying nature of response variation.

\section{AUTHOR NOTE}

This research was supported by Grant MH066855 to A.P.B. We thank Allen Neuringer, Cynthia Fast, Dennis Garlick, and Jared Wong for comments on a draft of the manuscript. We thank Alvin Chan, Tania Hioe, Andrea Miller, Charlton Ong, and Bryan Park for their assistance in conducting experimental sessions. Correspondence concerning this article should be addressed to A. P. Blaisdell, Department of Psychology, UCLA, 1285 Franz Hall, Box 951563, Los Angeles, CA 90095-1563 (e-mail: blaisdell@psych.ucla.edu). 


\section{REFERENCES}

Antonitis, J. (1951). Response variability in the white rat during conditioning, extinction, and reconditioning. Journal of Experimental Psychology, 42, 273-281.

AtNIP, G. W. (1977). Stimulus- and response-reinforcer contingencies in autoshaping, operant, classical, and omission training procedures in rats. Journal of the Experimental Analysis of Behavior, 28, 59-69.

Balsam, P. D., Deich, J. D., Ohyama, T., \& Stokes, P. D. (1998). Origins of new behavior. In W. O'Donohue (Ed.), Learning and behavior therapy (pp. 403-420). Boston: Allyn \& Bacon.

Bizo, L. A., Bogdanov, S. V., \& Killeen, P. R. (1998). Satiation causes within-session decreases in instrumental responding. Journal of Experimental Behavior: Animal Behavior Processes, 24, 439-452.

Brown, P. L., \& Jenkins, H. M. (1968). Auto-shaping of the pigeon's key-peck. Journal of the Experimental Analysis of Behavior, 11, $1-8$.

Cherot, C., Jones, A., \& Neuringer, A. (1996). Reinforced variability decreases with approach to reinforcers. Journal of Experimental Psychology: Animal Behavior Processes, 22, 497-508.

DeMarse, T. B., Killeen, P. R., \& BaKer, D. (1999). Satiation, capacity, and within-session responding. Journal of the Experimental Analysis of Behavior, 72, 407-423.

ECKERMAN, D. A., \& LANSON, R. N. (1969). Variability of response location for pigeons responding under continuous reinforcement, intermittent reinforcement, and extinction. Journal of the Experimental Analysis of Behavior, 12, 73-80.

Gharib, A., Derby, S., \& Roberts, S. (2001). Timing and the control of variation. Journal of Experimental Psychology: Animal Behavior Processes, 27, 165-178.

Gharib, A., Gade, C., \& Roberts, S. (2004). Control of variation by reward probability. Journal of Experimental Psychology: Animal Behavior Processes, 30, 271-282.

Herrick, R. M., \& Bromberger, R. A. (1965). Lever displacement under a variable ratio schedule and subsequent extinction. Journal of Comparative \& Physiological Psychology, 59, 392-398.

Millenson, J. R., \& Hurwitz, H. M. B. (1961). Some temporal and sequential properties of behavior during conditioning and extinction. Journal of the Experimental Analysis of Behavior, 4, 97-106.

NeURINGER, A. (1991). Operant variability and repetition as functions of interresponse time. Journal of Experimental Psychology: Animal Behavior Processes, 17, 3-12.

Neuringer, A., Kornell, N., \& Olufs, M. (2001). Stability and variability in extinction. Journal of Experimental Psychology: Animal Behavior Processes, 27, 79-94.
Notterman, J. M. (1959). Force emission during bar pressing. Journal of Experimental Psychology, 58, 341-347.

PAge, S., \& Neuringer, A. (1985). Variability is an operant. Journal of Experimental Psychology: Animal Behavior Processes, 11, 429-452.

Pavlov, I. P. (1927). Conditioned reflexes (G. V. Anrep, Trans.). London: Oxford University Press.

Pinheiro, J. C., \& Bates, D. M. (2004). Mixed-effects models in S and $S$-PLUS. New York: Springer.

Rescorla, R. A., \& Solomon, R. L. (1967). Two-process learning theory: Relationships between Pavlovian conditioning and instrumental learning. Psychological Review, 74, 151-182.

SchWARTZ, B. (1982). Interval and ratio reinforcement of a complex sequential operant in pigeons. Journal of the Experimental Analysis of Behavior, 37, 349-357.

SKINNER, B. F. (1948). "Superstition" in the pigeon. Journal of Experimental Psychology, 28, 168-172.

SPENCE, K. W. (1956). Behavior theory and conditioning. New Haven, CT: Yale University Press.

Stahlman, W. D., Roberts, S., \& Blaisdell, A. P. (2010). Effect of reward probability on spatial and temporal variation. Journal of Experimental Psychology: Animal Behavior Processes, 36, 77-91.

Stebiins, W. C., \& Lanson, R. N. (1962). Response latency as a function of reinforcement schedule. Journal of the Experimental Analysis of Behavior, 5, 299-304.

TRAPOLD, M. A., \& Overmier, J. B. (1972). The second learning process in instrumental learning. In A. H. Black \& W. F. Prokasy (Eds.), Classical conditioning II: Current research and theory (pp. 427-452). New York: Appleton-Century-Crofts.

Williams, D. R., \& Williams, H. (1969). Auto-maintenance in the pigeon: Sustained pecking despite contingent nonreinforcement. Journal of the Experimental Analysis of Behavior, 12, 511-520.

\section{NOTE}

1. Our statistical analysis assumes a continuous, not discrete, dependent variable. Given that technology limitations produced discrete values, we added uniform variability to these values to span the gaps (i.e., values were jittered such that each could be increased by as much as half of the distance to the adjacent value). The technique produced smoother fits of the distributions without changing the fundamental nature of these distributions. This approach was deemed necessary to identify the nature of the distribution so that we could find the best transformation to normality.

(Manuscript received May 14, 2009; revision accepted for publication October 10, 2009.) 